\title{
Design and status of the Mu2e experiment
}

\author{
Stefano Miscetti ${ }^{1}$ a on behalf of the Mu2e Collaboration \\ ${ }^{1}$ Laboratori Nazionali di Frascati dell'INFN, Via Enrico Fermi 40, 00044, Frascati, Italy
}

\begin{abstract}
The Mu2e experiment aims to measure the charged-lepton flavor violating neutrino-less conversion of a negative muon into an electron in the field of an aluminum nucleus. The objective is to improve the previous measurement by four orders of magnitude with the use of a similar technique. For the conversion process, the signal will appear as a mono-energetic electron very close to the muon rest mass. In the Standard Model these process have negligible rates. However, in many Beyond the Standard Model scenarios their rates are within the reach of next generation experiments. In this paper, we explain the sensitivity to new physics scale and the complementarity of approach and reach provided by MU2E with respect to Mu3e and MEG upgrade. Mu2e experimental technique, design and status will be the focus of this paper.
\end{abstract}

\section{Introduction}

In the Standard Model, SM, hadronic flavor transitions are allowed and the CKM matrix represents the probability for a quark of a specific family to turn into a different quark of another family. In the lepton sector, since SM neutrinos are massless, transitions between charged and neutral leptons preserve flavor. Nevertheless, flavor transitions between neutral leptons have been observed in neutrino oscillations that imply non conservation of lepton flavor number. Even assuming massive neutrinos, charged lepton flavor violation (CLFV) processes are extremely suppressed in the SM. The SM constraint on the branching ratio for $\mu \rightarrow e \gamma$ is $B(\mu \rightarrow e \gamma) \leq 10^{-52}$ [1]. Theories beyond the Standard Model, BSM, predict additional particles and interactions that can enhance such processes up to a measurable level (for a recent review see [2]).

Experimentally, the search for CLFV has a long history both in the muon and tau decays $(\mu \rightarrow e \gamma, \mu \rightarrow 3 e$, $\tau \rightarrow \mu \gamma, \tau \rightarrow 3 \mu$ ) as well as muon conversion processes, $\mu N \rightarrow e N$, in the presence of a nucleus. Starting with the pioneering measurements of cosmic rays in the late 1940's, the sensitivity has improved by many orders of magnitude and reached stringent limits for both $\mu \rightarrow \mathrm{e \gamma}$ (with $\mathrm{BR}<5.7 \times 10^{-13}$ at $90 \%$ C.L. [3]) and muon conversion on gold $\left(R_{\mu e}<6 \times 10^{-13}\right.$ [4]). An international search of CLFV continues today with the completion of MEG, its upgrade MEG-II and the proposed Mu3e search at PSI as well as the searches for muon to electron conversion with Mu2e at FNAL and COMET at J-PARC [5]. CLFV searches are of particular interest because of the possibility to carry out clean measurements free of theoretical background. In the case of observation they indicate a clear evidence of physics beyond the standard model while

\footnotetext{
a e-mail: Stefano.Miscetti@LNF.INFN.IT
}

in the case of non observation they pose strong limits on the development of new theories.

A model independent description of the CLFV transitions for physics beyond the Standard Model is provided by an effective lagrangian [6] where the different processes are divided in dipole amplitudes and four-fermion, or contact term, operators. The $\mu \rightarrow$ e $\gamma$ decay is mainly sensitive to the dipole amplitude, while $\mu \rightarrow e$ conversion and $\mu \rightarrow 3 e$ receive contributions also from the fourfermion interactions. It is customary to parametrize the interplay between the two amplitudes by means of two parameters [6]: $\Lambda$, which sets the mass scale and $\kappa$, which governs the ratio of the four-fermion to the dipole amplitude. For $k<<1(>>1)$ the dipole-type (four-fermion) operator dominates. Figure 1 summarizes the power of different searches to explore this parameter space. A scale $\Lambda<700 \mathrm{TeV}$ is already excluded by present limits and poses serious constraints on Standard Model extensions, primarily, supersymmetry. Nevertheless, $\Lambda$ is only an effective scale and is not immediately proportional to the mass of new particles accessible by direct $\mathrm{TeV}$ scale searches at LHC. The interpretation of an eventual direct observation of new physics at LHC will have to take into account precise measurements from MEG and Mu2e where the comparison between these determinations will provide the constraints necessary in pinning down the underlying theory.

An example of interplay between MEG and Mu2e is present in the scalar leptoquark searches. In Fig. $2 a$, the reach for the new coupling $\lambda$, as defined from eq. 14 of [7], is reported for a range of scalar leptoquark masses for both MEG upgrade and conversion experiments. The compilation is extracted from the Mu2e TDR. It is clear that while not excluding the Leptoquark existence at few $\mathrm{TeV}$ masses, the CLFV coverage extends up to masses of $\mathrm{O}($ $100 \mathrm{TeV}$ ). Another example is provided by the left-right 


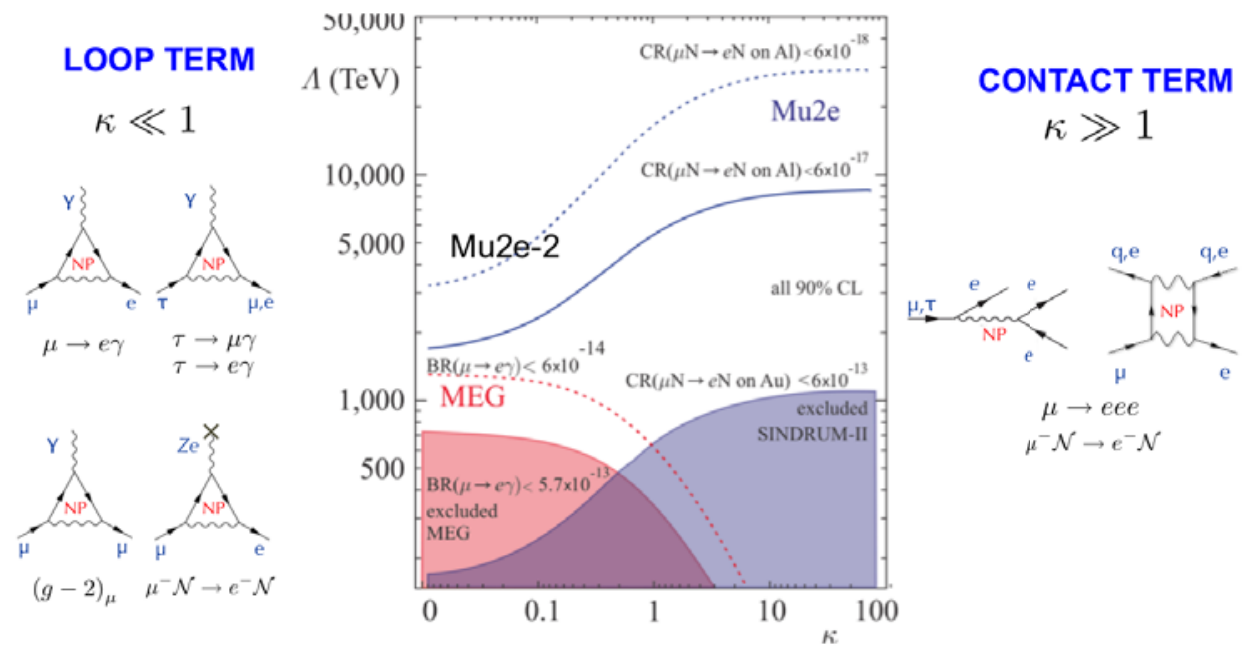

Figure 1. Sensitivity of $\mu \rightarrow e \gamma, \mu \rightarrow e$ transition and $\mu \rightarrow 3 e$ to the scale of new physics $\Lambda$ as a function of the parameter $\kappa$. The shaded areas are excluded by present limits. On the left (right) side, the dipole (four-fermion) diagrams are shown for the different CLFV processes.

(a)

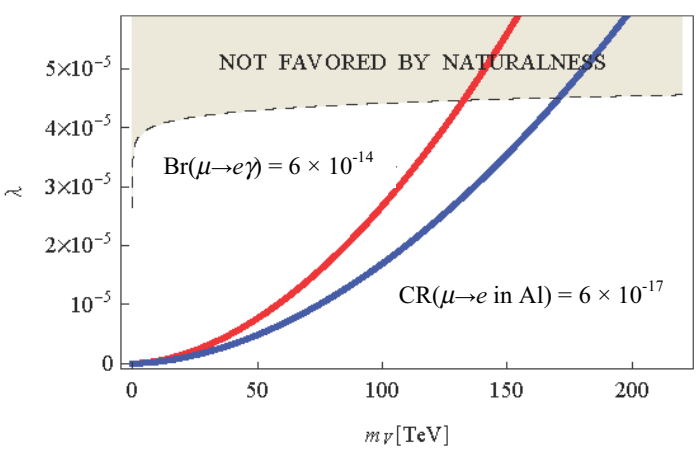

(b)

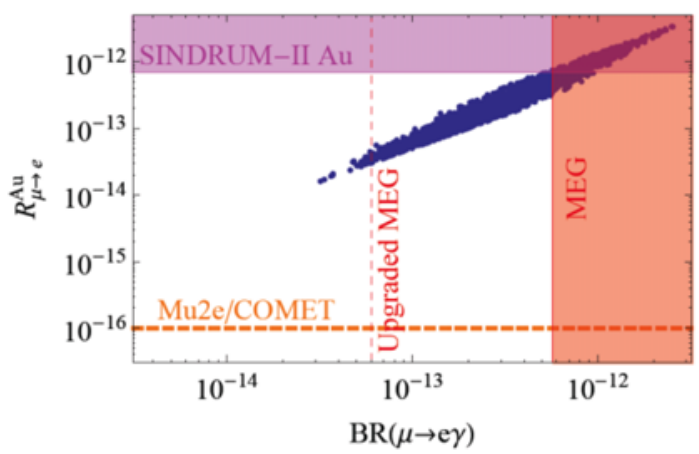

Figure 2. (a) Reach acheavable in scalar leptoquark search for Upgraded MEG and Mu2e as a function of the coupling $\lambda$ and the scalar leptoquark mass. (b) Expected BR and $R_{\mu e}$ for Left-Right symmetric models for Upgraded MEG and muon conversion experiments.

symmetric models. A recent study [8] predicts the CLFV rates in this environment assuming a new mass breaking scale at around $5 \mathrm{TeV}$. The correlation between the BR for the MEG upgrade and $R_{\mu e}$ for the conversion experiments are shown in Fig. $2 b$. It is clear that the two experiments can cover the expectations for the full phase space of this theory and help constraining it.

\section{Mu2e: search for $\mu$ to $e$ conversion at FNAL}

In presence of a nucleus, the measurement of the neutrinoless $\mu^{-}$to $\mathrm{e}^{-}$conversion rate is defined relatively to the ordinary capture of the muon on the nucleus as follows:

$$
\left.R_{\mu e}=\frac{R\left(\mu^{-}+A(Z, N) \rightarrow e^{-}+A(Z, N)\right)}{R\left(\mu^{-}+A(Z, N) \rightarrow v_{\mu}+A(Z-1, N)\right.}\right) .
$$

The conversion signal, $\mathrm{CE}$, consists of a monoenergetic electron (see Fig. 3(left)), close to the muon mass and corrected for binding energy and nuclear recoil, that has to be separated by means of high momentum resolution from the spectrum of the electrons produced by the muon decay in orbit process, DIO (see Fig. 3(right)). Stopped muons have a large chance of decaying when orbiting around the nucleus and their spectrum substantially differs from free decay due to the presence of a large recoil tail that falls rapidly as the energy approaches the kinematical endpoint. Unfortunately, in Sindrum-II additional background sources were dominating. In particular, after rejection of cosmics, a prompt background coming from radiative pion capture, $\mathrm{RPC}, \pi+N \rightarrow \gamma^{*}+N^{\prime}$, was found to be the most resistant background. In this process the electron positron pair, produced either by internal or external 

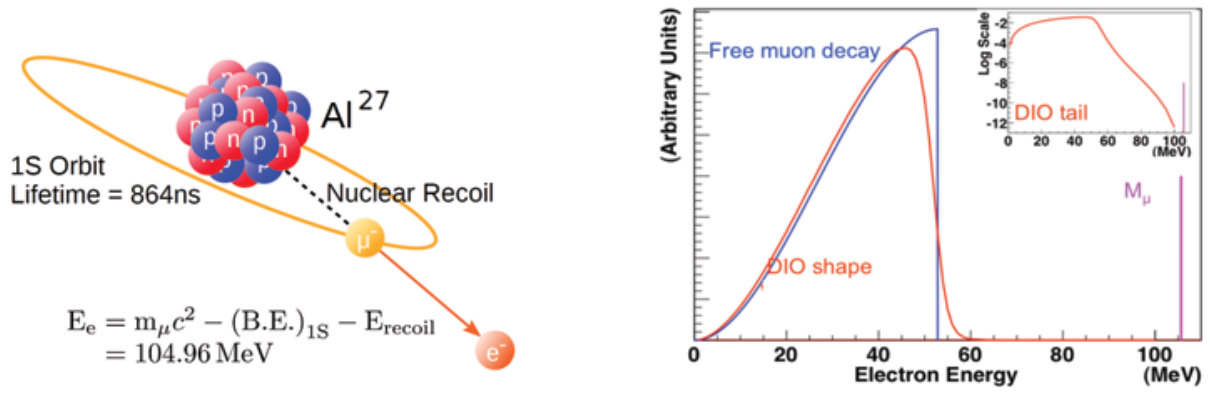

Figure 3. (Left) Sketch of the muon to electron conversion process. (Right) Energy spectrum for electrons produced from muon decays in orbit. The spectrum is a modified Michel spectrum with a long tail that falls quickly as $\left(E_{e}-E_{\text {endpoint }}\right)^{5}$ and comes in proximity to the kinematic endpoint. Perfect resolution is assumed.

conversion, becomes a source of CE candidate when the $\mathrm{e}^{-}$momentum gets in the right selection window. These observations have driven the design strategy of the new generation muon to electron conversion experiments like Mu2e. The Mu2e goal is to achieve a single CE event sensitivity of $2.5 \times 10^{-17}$, or constrain the $R_{\mu}$ value to below $6 \times 10^{-17}$ at $90 \%$ C.L., four orders of magnitude better than the previous limit.

There are four ingredients to achieve: high intensity, pulsed beam structure, high proton extinction and a precise detector. A high intensity muon beam. The goal is to increase by a factor of four the nowadays target muon intensity to $10^{11} / \mathrm{sec}$. This can be achieved by means of a high muon production and a curved solenoidal system to allow for charge and momentum selection. The solenoidal system creates a selective transport channel that increases intensity while reducing the associated need for beam power (compare $1 \mathrm{MW}$ at PSI for Sindrum-II and $8 \mathrm{~kW}$ for Mu2e). A pulsed beam structure allows for the rejection of the prompt background. The target has to be selected in a way that the muon lifetime, $\tau_{\mu}$, in the bound system is smaller than the bunch period. Mu2e utilizes an aluminum target ( $\tau_{\mu}=864 \mathrm{~ns}$ ) that well matches the bunch structure of the Fermilab accelarator complex. For the beam delivered to $\mathrm{Mu} 2 \mathrm{e}$ the micro-bunch structure occur in a window of 1684 ns. The trick here is to wait for the beam-flash of particles travelling with the transported beam to disappear and waiting for the most relevant prompt backgrounds to decay. The start of the data acquisition is typically set at 700 ns after the bunch arrival time. The effects of the unsynchronized particles traveling with the beam have to be negligible. This is referred to as the proton extinction requirement. Calculation by full simulation has shown that in order to suppress the prompt backgrounds generated by beam electrons, in-flight-decay muons, in-flight-decay pions and RPC requires a pulsed beam where the ratio of the beam between pulses to the beam contained in a pulse is below $10^{-10}$. A redundant high-precision detector has to analyze the captured products in order to separate $\mathrm{CE}$ and DIO spectra and reduce to negligible contribution the additional background sources.

\subsection{The Mu2e detector layout}

The layout of the Mu2e experiment is shown in Figure 4. An array of superconducting solenoids forms a graded magnetic system composed of a Production Solenoid, PS, a Transport Solenoid, TS, and a Detector Solenoid, DS.

The PS contains a tungsten target that intercepts an 8 $\mathrm{GeV}$ pulsed proton beam brought from the FNAL delivery ring by means of a dedicated beam-line. The gradient field in the PS increases from 2.5 to 4.6 Tesla in the same direction of the incoming beam and opposite to the outgoing muon beam direction. This gradient field works as a magnetic lens used to focus low energy particles into a transport channel. The focused beam is constituted by muons, pions and a small number of protons and antiprotons. The S-shaped Transport Solenoid efficiently transfers low energy, negatively charged particles to the end of a beam-line while allowing for a large fraction of the pions to decay into muons. A midsection collimator attenuates nearly all positively charged particles while the antiproton residual background is reduced by a special absorber. The DS presents a graded field from 2 to 1 Tesla in the upstream region where the stopping target resides. This lens increases the acceptance for CE while aiding in the rejection of beam-related backgrounds. A uniform magnetic field of 1 Tesla occupies the region of the tracker and calorimeter systems. Approximately $50 \%$ of the muon beam is stopped by the target while the remaining beam is dumped at the end of the cryostat. The DS is covered externally by a Cosmic Ray Veto system.

When muons stop in the aluminum target, they are captured in an atomic excited state and thus prompty fall to the ground state with $39 \%$ decaying in orbit while the remaining $61 \%$ are captured on the nucleus. Low energy photons, neutrons and protons are emitted in the nuclear capture process and constitute an environmental background that produces an ionization dose and a neutron fluence on the detection systems as well as an accidental occupancy for the reconstruction program. This background is fully simulated in overlap with the signals coming from the apparatus.

The tracking detector is composed of low mass straw drift tubes oriented transverse to the solenoid axis and con- 


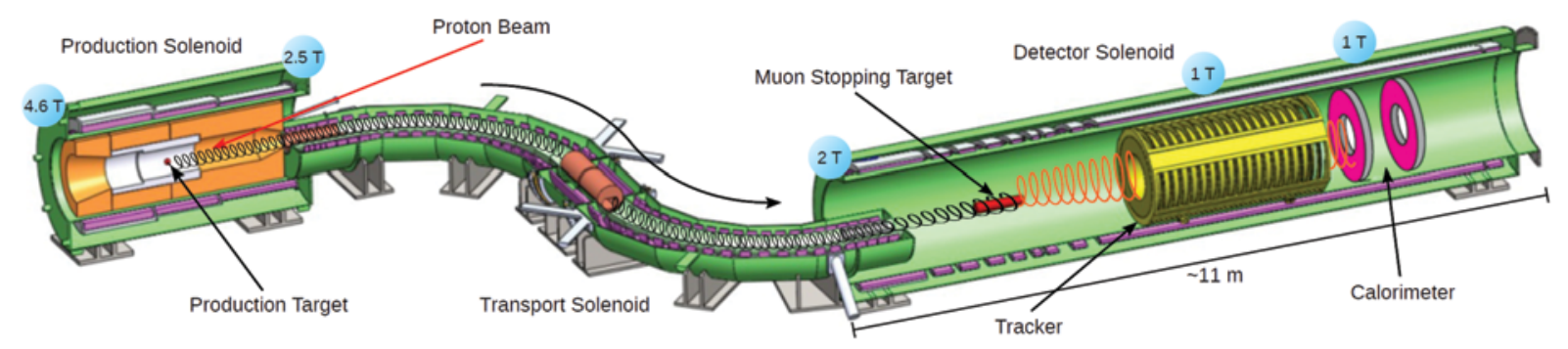

Figure 4. Layout of the Mu2e experiment.
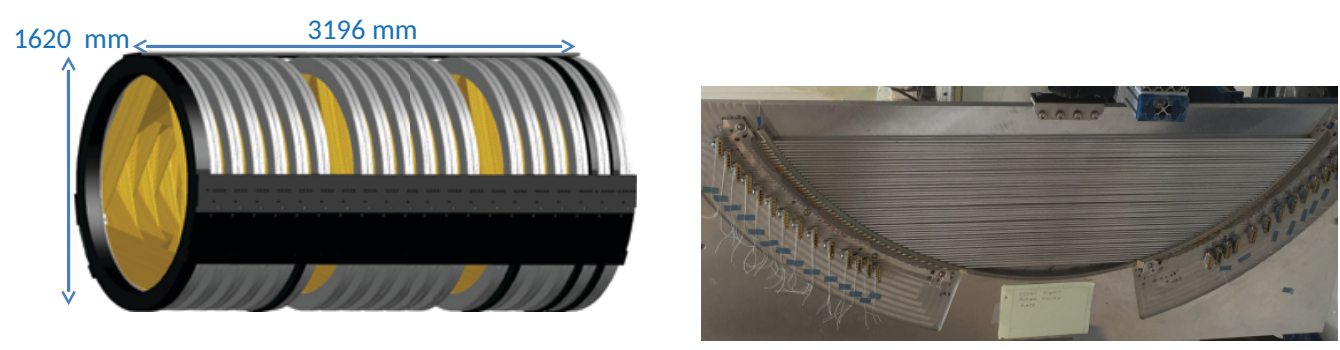

Figure 5. (Left) Sketch of the Mu2e straw tracker system. (Right) Picture of the first prototype built for straw tube panel.

sists of nearly 20000 straw tubes arranged in 18 station as shown in Fig. 5(left). Each tube is $5 \mathrm{~mm}$ in diameter and contains a $25 \mu \mathrm{m}$ sense wire. The straw walls are made out of Mylar and have a thickness of $15 \mu \mathrm{m}$. The tracker is around $3 \mathrm{~m}$ long and placed inside a $1 \mathrm{~T}$ uniform magnetic field. It measures the momenta of the charged particles from the reconstructed trajectories using the hits detected in the straw. A circular hole inside the tracker allows it to be insensitive to charged particle momenta below 55 $\mathrm{MeV} / \mathrm{c}$. The tracking preparation proceeds well as shown by the picture of the first straw-panel prototype built (see Fig. 5(right)).

The electromagnetic calorimeter system [9] is composed by two disks of scintillating crystals and is placed downstream of the tracker. A single disk is composed of $830 \mathrm{BaF}_{2}$ crystals readout by two solar blind $\mathrm{UV}$ extended avalanche photo-diodes (APD). In Fig. 6(left) a drawing of the disks is shown. Similarly to the tracker, the inner circular hole allows to be insensitive to the DIO electrons up to $55 \mathrm{MeV} / \mathrm{c}$ momenta. The calorimeter tasks are that of providing a powerful particle identification between muons and electrons, an independent trigger system and a seed for tracking in a complicated reconstruction environment. In order to fulfill these requirements the calorimeter must provide an energy resolution of $\mathrm{O}(5 \%)$, a time resolution $<500 \mathrm{ps}$ and a position resolution better than $1 \mathrm{~cm}$ for $100 \mathrm{MeV}$ electrons. The selected crystals should survive a dose of $100 \mathrm{krad}$ and a fluence of up to $10^{12} \mathrm{n} / \mathrm{cm}^{2}$. The photosensors are shielded by the crystals themselves and should only sustain a fluence of up to $3 \times 10^{11} \mathrm{n}_{1 \mathrm{MeV}} / \mathrm{cm}^{2}$. Prototypes for the solar blind APDs have been developed but the dark noise is too high and cooling them down to $-10^{\circ} \mathrm{C}$ is needed. Backup options are being explored.
One major background source for $\mathrm{Mu} 2 \mathrm{e}$ is related to cosmic ray muons producing false CE's when interacting with the detector materials. These events occur at a rate of approximately one/day. In order to reduce their contributions to below 0.1 event in the experiment lifetime the external area of the DS and a part of the TS are covered by a Cosmic Ray Veto, CRV, system. The requirement for the system is to obtain a vetoing efficiency of at least $99.99 \%$ for cosmic ray tracks while withstanding an intense radiation environment. Comprised of four staggered layers of scintillation counters (see 6(right)), the CRV utilizes two embedded Wavelength Shifting Fibers, each readout by means of $2 \times 2 \mathrm{~mm}^{2}$ Hamamatsu SIPM. Test beams on full size prototype have been carried out.

Further instrumentation exists, such as the monitor to measure the proton beam extinction and a High Precision Germanium Detector to monitor the stopping rate in the aluminum target. The latter one is needed to provide the normalization for the $R_{\mu e}$ conversion rate. Further information can be found in ref. [5] .

\subsection{Reconstruction of CE candidates}

At this energy the momentum resolution is dominated by fluctuations in the energy loss in the target caused by multiple scattering and bremsstrahlung in the tracker. By performing a full simulation of the tracker, a pattern recognition and a Kalman fitter allow us to obtain for the tracking a CE reconstruction efficiency of $9 \%$ for good quality tracks and a minimum of 25 hits/track. The resolution is well parametrized by a Crystal Ball function with a negative bremsstrahlung tail, a gaussian core of $116 \mathrm{keV}$ and a long exponential positive resolution tail. This re- 

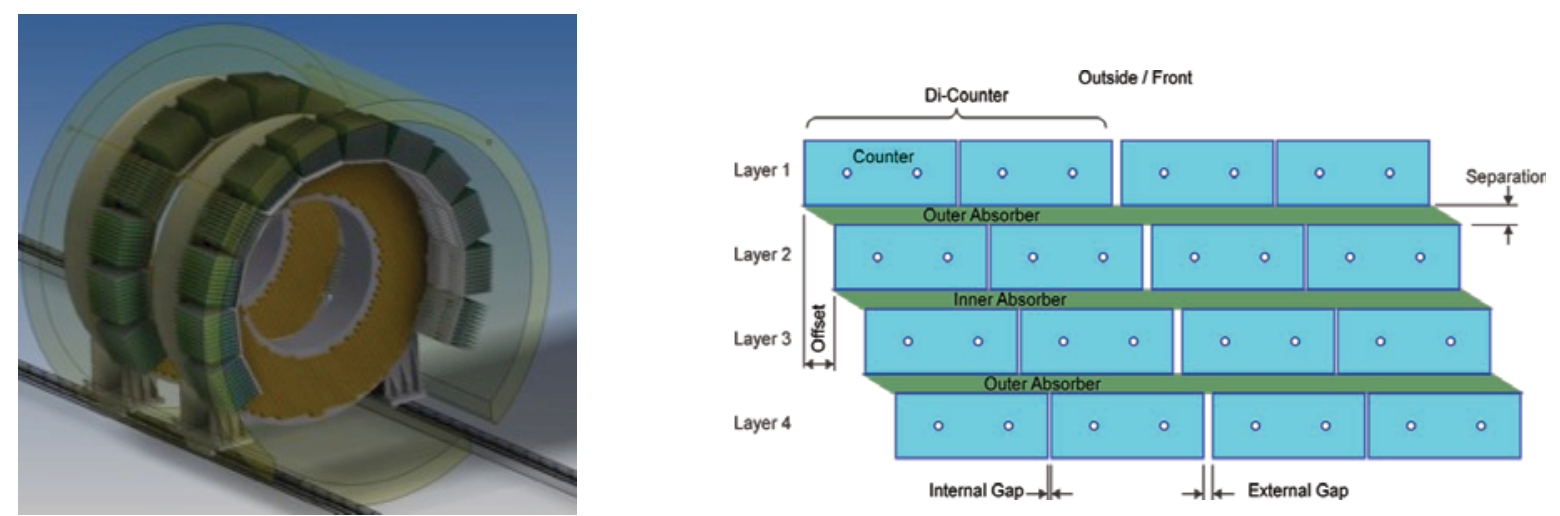

Figure 6. (Left) CAD drawings of the calorimeter disks. Calorimeter innermost (outermost) radius is of $351 \mathrm{~mm}(670 \mathrm{~mm}) \mathrm{with}$ a crystal length of $200 \mathrm{~mm}$. Layout of the FEE and digitization crates is also shown. (Right) Schematic drawing of the cross-sectional view of a CRV counter. Each counter is $20 \mathrm{~mm}$ thick with a width of 50 or $60 \mathrm{~mm}$ and a length up to $6.6 \mathrm{~m}$.

sult is obtained in a hostile environment due to the large quantity of neutrons, photons and protons produced from the muon capture processes. Note that the muon stopping rate needed to arrive to the final goal of $6 \times 10^{17}$ stopped muons is at around $10 \mathrm{GHz}$. In Fig. 7(left), an example of a single micro-bunch event in the tracker is shown. This has been obtained with a Geant-4 simulation displaying all hits generated by the environmental background. The irreducible background is provided by the DIO electrons that have to be distinguished by the mono-energetic CE. The finite tracking resolution and the positive reconstruction tail have a large effect on the falling spectrum of the DIO background that translates to a residual contamination in the signal region. Fig. 7(right) shows the signal and background distributions as seen by a full simulation of the experiment (pileup included) in the following conditions: (i) $3.6 \times 10^{20}$ proton on target (ii) a corresponding number of $6 \times 10^{17}$ muon stops and (iii) a $R_{\mu e}$ of $10^{-16}$. After maximizing the signal over the background the best selection corresponds to events counted in a momentum window between 103.75 and $105 \mathrm{MeV} / \mathrm{c}$. A DIO contribution of 0.199 events is estimated and 3.5 candidates are observed. Estimate of other backgrounds are presented in Tab.3 for a total background contribution of 0.37 events. This counting corresponds to set a limit on $R_{\mu e}$ below $6 \times 10^{-17}$ at $90 \%$ C.L. , in good agreement with the experiment design goal. A very important step for this measurement is the tracker calibration. To accurately predict the DIO rate, we should calibrate the tracker momentum i.e. determine the shifts, the scale and the resolution of the measured momentum compared to the true one. After a basic alignment, based on construction and installation survey data, an thorough calibration will be based both on the usage of the DIO sample and on independent processes of low energy two body decay, such as the one from stopped $\pi^{+}$on target undergoing the $\pi \rightarrow e v$ decay. Selecting positive pions will help avoid nuclear capture but asks for reversing the momentum selection in the TS. Moreover, the data have to be collected at shorter rate and an extrapolation from lower energies to the conversion endpoint is still needed.

\subsection{Experiment status and schedule}

At the moment of writing the Mu2e experiment has succesfully completed the CD-2 review for all systems and the CD-3 reviews for the superconducting cables, civil construction and the Transport Solenoids.

The heart of the Mu2e apparatus is provided by a superconducting magnetic system whose design, fabbrication, assembly and commissioning drives the experiment schedule. The status of the magnet as well as the construction and testing of the superconducting cables is satisfactory. An international bid for the DS and PS has been concluded and the construction phase for the large magnets are started at General Atomics, San Diego, USA. For the TS, a prototype of one module has been built by ASG Superconducting in Genova in collaboration with the INFN group of Genova. Tests and quality control of the prototype have been carried out this summer with satisfactory results. The bid on the TS has also been completed and has been assigned to the ASG. Since the ground breaking cerimony for the experimental hall, held on April 2015, the civil construction is proceeding at a rapid pace. Upon completion of the CD-3 review expected for the summer of 2016, the construction of the detectors and accelerator will begin. The schedule foresees a completion of the installation and commissioning with beam at the end of 2020 .

\section{Conclusions and perspectives}

The Mu2e experiment design and construction proceeds well and iis on schedule to be commissioned with a beam by the end of 2020. Its goal is to probe CLFV with a single event sensitivity of $2.5 \times 10^{-16}$ or set an upper limit on the conversion rate $<6 \times 10^{-17}$ at $90 \%$ C.L., thus improving the previous sensitivity by four orders of magnitude. For the long term future $(>2025)$ the possibility of a Mu2e phase- 2 is being explored with the goal of increasing the sensitivity by an additional factor of 10 . This can be obtained with a higher beam intensity and a detector capable of handling the the accidental activity occuring from muon 

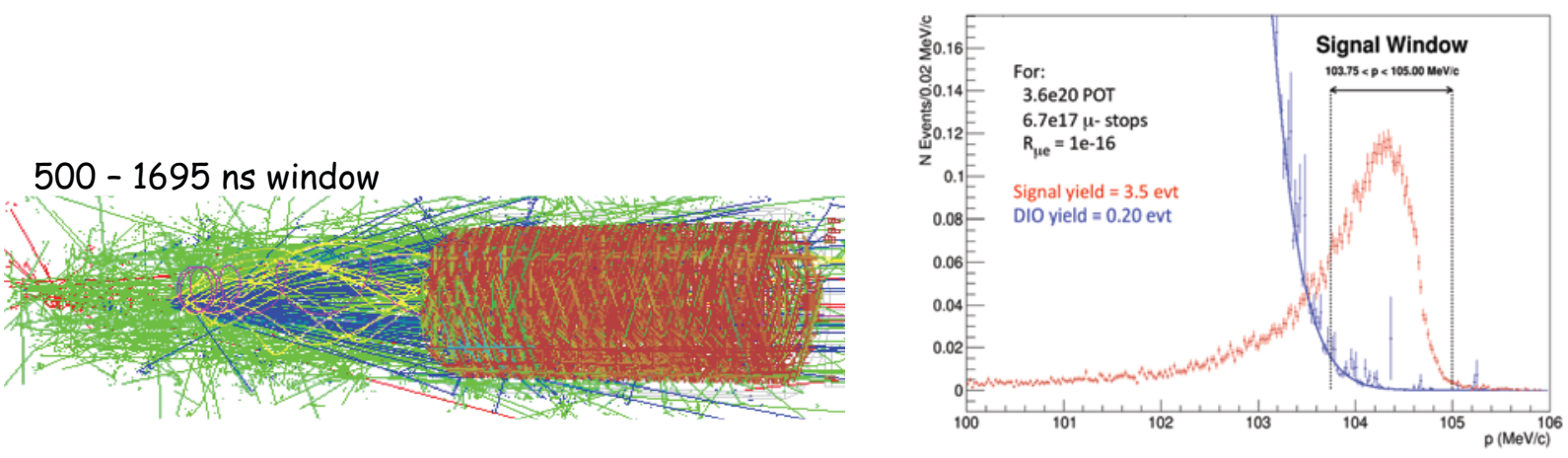

Figure 7. (Left) event display of a single micro-bunch event. (Right) full simulation of DIO and CE events for an assumed $R_{\mu e}$ of $10^{-16}$.

\begin{tabular}{lll}
\hline Category & Background process & Estimated Yield \\
\hline Intrinsic & Decay in orbir (DIO) & $0.199 \pm 0.092$ \\
& Muon Capture (RMC) & $0.000 \pm 0.004$ \\
Late Arriving & Pion capture (RPC) & $0.023 \pm 0.006$ \\
& Muon decay in flight & $<0.003$ \\
& Pion decay in flight & $0.001 \pm 0.001$ \\
& beam electrons & $0.003 \pm 0.001$ \\
Miscellaneous & pbar & $0.047 \pm 0.024$ \\
& Cosmic rays & $0.092 \pm 0.020$ \\
\hline & Total & $0.37 \pm 0.10$
\end{tabular}

Talents Plan of China; the Helmholtz Association of Germany; and the EU Horizon 2020 Research and Innovation Progam under the Marie Sklodowska-Curie Grant Agreement N.690385. Fermilab is operated by Fermi Research Alliance, LLC under Contract No. De-AC02-07CH11349 with the US Department of Energy.

\section{References}

[1] S.M.Bilenky et al., Phys. Lett. B67, 309 (1977)

Table 1. Expected background list as evaluated by full simulation. Extracted from the Mu2e TDR.

[2] F.Grancagnolo et al., Nucl.Phys.Proc.Supp. 248-250 1-146 (2014).

[3] The MEG collaboration,Phys.Rev.Lett. 110201801 (2013).

capture. In this context only an option based on the new

[4] The SINDRUM II Collaboration, Eur.Phys.J. C47 337-346 (2006)

Proton Improvement Plan, PIP-2, is being studied.

\section{Acknowledgments}

We are grateful for the vital contributions of the Fermilab staff and the technical staff of the participating institutions. This work was supported by the US Department onf Energy; the Italian Istituto Nazionale di Fisica Nucleare; the US National Science Foundation; the Ministry of Education and Science of the Russian Federation; the Thousand

[5] The Mu2e Collaboration, FERMILAB-TM-2594, ArXiv:1501.05241 (2015).

[6] A. De Gouvea et al., Prog. Part. Nucl. Phys 71 75-92 (2013).

[7] J.M.Arnold et al., Phys.Rev D88 035009 (2013).

[8] C.H.Lee et al., Phys.Rev D88 092010 (2013).

[9] X.. et al., Nucl. Instr. and Meth. A5857, 10.1016/j.nima.2015.09.074. 